\title{
Correlation between cosmic noise absorption and VHF coherent echo intensity
}

\author{
R. A. Makarevitch ${ }^{1, *}$ and F. Honary ${ }^{1}$ \\ ${ }^{1}$ Department of Communication Systems, Lancaster University, Lancaster, LA1 4WA, UK \\ *now at: Department of Physics, La Trobe University, Victoria, 3086, Australia
}

Received: 27 September 2004 - Revised: 7 January 2005 - Accepted: 12 January 2005 - Published: 27 July 2005

Part of Special Issue "Atmospheric studies by optical methods"

\begin{abstract}
We present examples and statistical analysis of the events with statistically significant correlation between the cosmic noise absorption (CNA) and the signal-to-noise ratio (SNR) of the VHF coherent echo intensity in the area monitored simultaneously by an imaging riometer and two oblique-sounding coherent VHF radars in Northern Scandinavia. By only considering the observations from the narrow riometer beams comparable (in terms of the intersection with the ionosphere) with the VHF radar cells, we identify $\sim 200$ one-hour high correlation periods (HCPs) for 2 years near the solar cycle maximum, 2000-2001. The HCP occurrence is maximized in the afternoon (12:00-17:00 UT, $\mathrm{MLT} \cong \mathrm{UT}+3)$, with the secondary peak near the midnight (21:00-02:00 UT). Relative to the VHF echo occurrence, HCPs occur more frequently from 11:00 to 20:00 UT. The diurnal variation of HCP occurrence is similar to that of the 1-h intervals with the lowest mean absorption $A<0.25 \mathrm{~dB}$.
\end{abstract}

The HCPs are observed more frequently during the winter months, which, combined with the fact that VHF echoes observed during HCPs exhibit features typical for field-aligned E-region irregularities, makes their association with the polar mesospheric echoes (for which some positive CNA/SNR correlation has been reported in the past) very unlikely. Instead, we attribute the high positive CNA/SNR correlation to the synchronous, to a first approximation, variation of the particle fluxes for two different but close sets of energies.

By considering the dependence of the CNA/SNR correlation coefficients for both VHF radars $\left(C_{A 1}\right.$ and $\left.C_{A 2}\right)$ upon the correlation between SNRs for two radars $\left(C_{12}\right)$, we show that both coefficients, $C_{A 1}$ and $C_{A 2}$, and the agreement between them decrease drastically with a $C_{12}$ decrease, which we interpreted through the progressively increasing role of the spatial inhomogeneity of the processes leading to the enhanced CNA and SNR. In this situation, a similarity between the radio signal collection areas should become important, and we demonstrate that the HCP occurrence and mean cor-

Correspondence to: R. A. Makarevitch

(r.makarevitch@lancaster.ac.uk) relation coefficient decrease as the riometer beams and radar cells become less comparable in terms of mutual orientation and closeness between the points of maximum sensitivity.

Keywords. Ionosphere (Auroral ionosphere; Particle precipitation; Instruments and techniques)

\section{Introduction}

Since the introduction of relative ionospheric opacity meters (riometers) in the late 1950s, cosmic noise absorption (CNA) measurements have been routinely used for studies of the Earth's ionosphere as a proxy for energetic particle precipitation at D-region altitudes $(60-90 \mathrm{~km})$, as well as at higher E- and F-region altitudes (see, for example, review papers by Hargreaves, 1969; Stauning, 1996a,b).

Studies have identified several types of absorption events according to the time of observations, event duration, shape of absorption intensity variation with time, accompanying phenomena and associated mechanisms. Thus, both midnight and morning auroral absorption have been attributed to the energetic (10-100 keV) electrons injected into the region of closed field lines that either immediately precipitate in the midnight sector or drift and precipitate further in the morning sector (Ansari, 1964). The same mechanism has been applied later to the daytime absorption (e.g. Nishino et al., 1999; Kavanagh et al., 2002).

Pre- and post-midnight auroral absorption have been found to exhibit some association with various optical auroral forms (see Hargreaves, 1969, and references therein), with the correlation coefficient between optical intensity and CNA maximized in the midnight sector and decreasing with time (Ansari, 1964). Absorption intensity has also been shown to correlate well with the $\mathrm{X}$-ray counting rate at $\sim 30 \mathrm{~km}$ (Brown and Barcus, 1963) and the magnetic disturbance level associated with the electrojet currents at 100$110 \mathrm{~km}$ measured by magnetometers (Brown and Campbell, 1962; Hargreaves, 1966; Kavanagh et al., 2004). Positive 


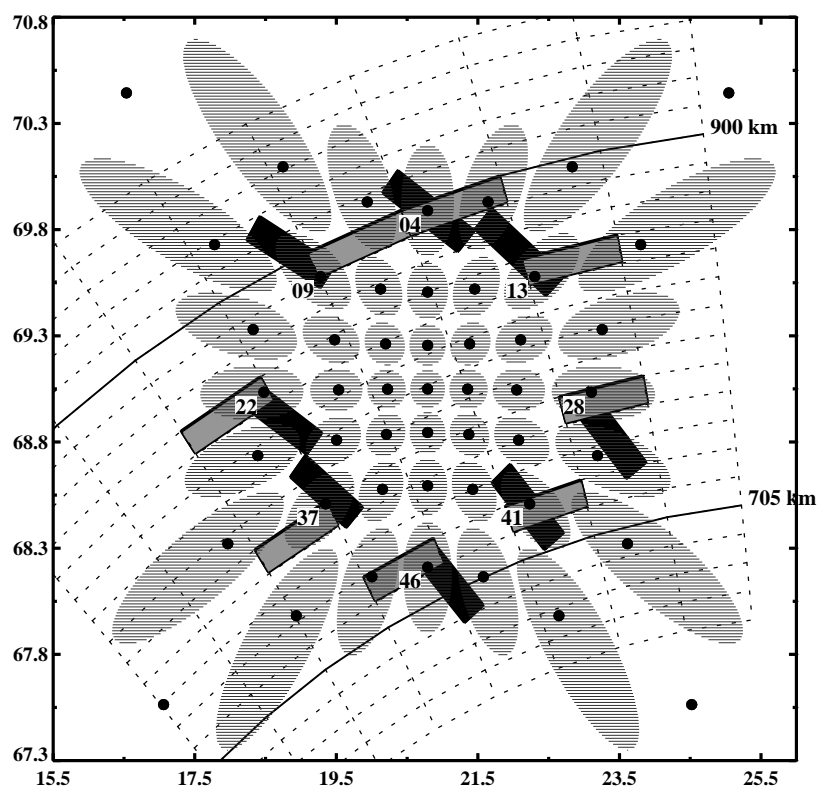

Fig. 1. The experimental setup in Northern Scandinavia showing the central positions of IRIS beams at $90 \mathrm{~km}$ by black circles, projections of the 3-dB IRIS beams by contours filled with horizontal lines, STARE Finland radar cells near the IRIS FoV by dotted contours, and STARE Finland (grey) and Norway (black) radar cells closest to the selected IRIS beams with digits nearby indicating the IRIS beam number.

correlation has been reported between the CNA and Polar Mesospheric Summer Echo (PMSE) intensity (Czechowsky et al., 1989; Kirkwood et al., 1995; Klostermeyer, 1999; Barabash et al., 2002) as observed by VHF radars, although statistically the correlation appears to be rather weak (Czechowsky et al., 1989; Bremer et al., 2000; Barabash et al., 2002).

In this paper we attempt to advance our knowledge of the processes leading to the enhanced cosmic noise absorption by exploring the relationship between the riometer absorption and backscatter power of auroral echoes detected by a VHF coherent radar. We consider the correlation between signal-to-noise ratio (SNR) of the VHF echoes and the CNA, and identify the periods of statistically significant high correlation or high correlation periods (HCPs) with correlation coefficients above 0.4 for 1 -min resolution data. We also identify the extended periods (over $3 \mathrm{~h}$ ) of very high correlation $(>0.8)$ and analyze statistically the HCP occurrence for a 2-year observational period. By employing the data from several close but spatially separated locations (beams of an imaging riometer), we study the effects on the correlation arising from the differences in collecting areas between riometer and VHF radar.

\section{Experiment setup}

The Lancaster University Imaging Riometer for Ionospheric Studies (IRIS) at Kilpisjärvi, Finland $\left(69.1^{\circ} \mathrm{N}, 20.8^{\circ} \mathrm{E}\right.$, $\sim 66^{\circ} \mathrm{MLAT}$ ) has been providing the data on the CNA at $38.2 \mathrm{MHz}$ for 49 directions since September 1994 (Browne et al., 1995). The riometer is analogous technically and operationally to other imaging riometers developed in the late 1980s by the University of Maryland group (see Detrick and Rosenberg, 1990, for details on imaging riometer techniques). Figure 1 shows the central positions or foci of the 49 beams and 3-dB contours at $90 \mathrm{~km}$ (the corner beam contours are not shown) of the IRIS by black dots and areas filled with horizontal lines, respectively.

The IRIS facility is conveniently located within the viewing areas of several other facilities in Northern Scandinavia, including the Scandinavian Twin Auroral Radar Experiment (STARE) Norway and Finland VHF radars $(\sim 140$ and $\sim 144 \mathrm{MHz})$ located in Midtsandan, Norway $\left(63.7^{\circ} \mathrm{N}\right.$, $\left.10.73^{\circ} \mathrm{E}\right)$ and Hankasalmi, Finland $\left(62.3^{\circ} \mathrm{N}, 26.6^{\circ} \mathrm{E}\right)$, looking roughly towards magnetic east and north, respectively. The E-region echoes are monitored in 8 beams separated by $3.6^{\circ}$ in azimuth ( $3.2^{\circ}$ half-power beam width) from 495 to $1245 \mathrm{~km}$ at $15-\mathrm{km}$ resolution. The data are integrated over 20 s. We show in Fig. 1, by dotted lines, the STARE Finland radar cells closest to the IRIS field of view (FoV). In the standard STARE mode (we only use such data), the single-pulse data are used to determine the power of the echoes and the double-pulse data are used to determine the Doppler velocity (Greenwald et al., 1978; Nielsen, 1982). The STARE echoes that had low power $(<1 \mathrm{~dB})$ were not considered.

In this study we only used the data from those IRIS beams whose half-power collection areas are comparable to the STARE radar cells (we thus did not consider either the central or the corner IRIS beams) and we show in Fig. 1 the radar cells closest to the selected IRIS beams for both Norway and Finland radars by black and grey areas, respectively, with digits showing the IRIS beam number.

\section{Observations}

One can easily notice from Fig. 1 that the degree of similarity between the IRIS and STARE collection areas depends not only on their size but also on the relative orientation and the distances between the points of maximum sensitivity/foci. The latter are simply the centers of the radar cells for STARE, while for IRIS these are the central positions of the riometer beams shown by large black dots. For example, IRIS beam 41 is very similar to the closest STARE Norway radar cell both in terms of orientation and distance between the foci. Both the STARE Finland focus and the centre of the intersection area of the STARE Norway and Finland radar cells are also close to the IRIS focus point. One can conclude that a comparison between the CNA intensity and the STARE backscatter powers would be more meaningful for IRIS beam 41 than, say, for beam 9 , for which all three foci are somewhat apart from each other, so that the measurements refer to different (although close) locations.

In the following two sections (Sects. 3.1 and 3.2) we perform a statistical analysis of the CNA/SNR correlation using 
the data from the IRIS beam 41 and corresponding STARE cells for a 2-year period of high solar activity, from January 2000 to December 2001, during which the STARE radars regularly observed the ionospheric echoes. Our aim is to explore diurnal and seasonal (if any) variations of the periods with statistically significant correlation. In this study we used the data that have been post-integrated with a period of $1 \mathrm{~min}$. This has been done simply to reduce the database size and computation time. It was found that the use of 20-s resolution data does not change the results of analysis in any significant way, with exception of slightly lower (by $\sim 10 \%$ ) resulting correlation coefficients.

For each 1-h period in 2000-2001, the linear Pearson correlation coefficients were computed between the IRIS absorption $A$ in $\mathrm{dB}$ and backscatter powers (SNRs, also in $\mathrm{dB}$ ) recorded by the STARE Norway and Finland radars $\left(C_{n a}\right.$ and $C_{f a}$, respectively), as well as the correlation between the STARE Norway and Finland powers $\left(C_{n f}\right)$. The correlation coefficients were entered into the database only if all three parameters had finite values (data were available) for at least $60 \%$ of the time within a given $1-\mathrm{h}$ period.

Figure 2 shows two examples of events with very high correlation $\left(C_{n a}>0.8\right.$ for over three hours) for (a) 12 and (b) 14 February 2000 . The CNA intensity $A$ is shown by the red line, while the STARE Norway (Finland) backscatter power variation is shown by the blue (green) line. The correlation levels $C_{n a}$ and $C_{f a}$ for each 1-h period of continuous ( $60 \%$ or more) observations are shown by the blue and green horizontal lines, respectively, with the correlation values shown by digits above the lines and by heights from the bottom of each panel (100\%-correlation period would be at the top; negative correlation periods are of the same height as the positive ones). Also shown in Fig. 2, by the dotted horizontal line, are the 0.4 correlation levels, comfortably above $99 \%$ confidence levels for both 60 - and 36-point $(60 \% * 60)$ time series (0.27 and 0.35).

One can notice that during the first event (a) the correlation is high $\left(C_{n a}, C_{f a}>0.4\right)$ for $6 \mathrm{~h}(11: 00-17: 00 \mathrm{UT})$ and it is very high $\left(C_{f a}>0.67, C_{n a}>0.84\right)$ for $3 \mathrm{~h}(14: 00-17: 00 \mathrm{UT})$. A similar pattern is observed for the second event (b), except that the very high correlation period is observed earlier (12:00-15:00 UT). Interestingly, the correlation with the Norway power appears to be generally greater. This can be seen not only from the correlation coefficients themselves but also from the simple comparison of the line plots, thus at $\sim 12: 30$ UT on 14 February 2000 the Norway SNR peaks almost simultaneously with the CNA, while Finland SNR variation only shows a broad maximum around this time.

\subsection{High correlation periods occurrence}

The correlation coefficients computed, as described above, are used in this section to investigate the diurnal and seasonal dependencies of the high correlation period (HCP) occurrence. In Fig. 3 each line corresponds to a HCP (both $C_{n a}$ and $C_{f a}>0.4$ ). The horizontal position of the line shows the time of day while the vertical position shows the time of year,

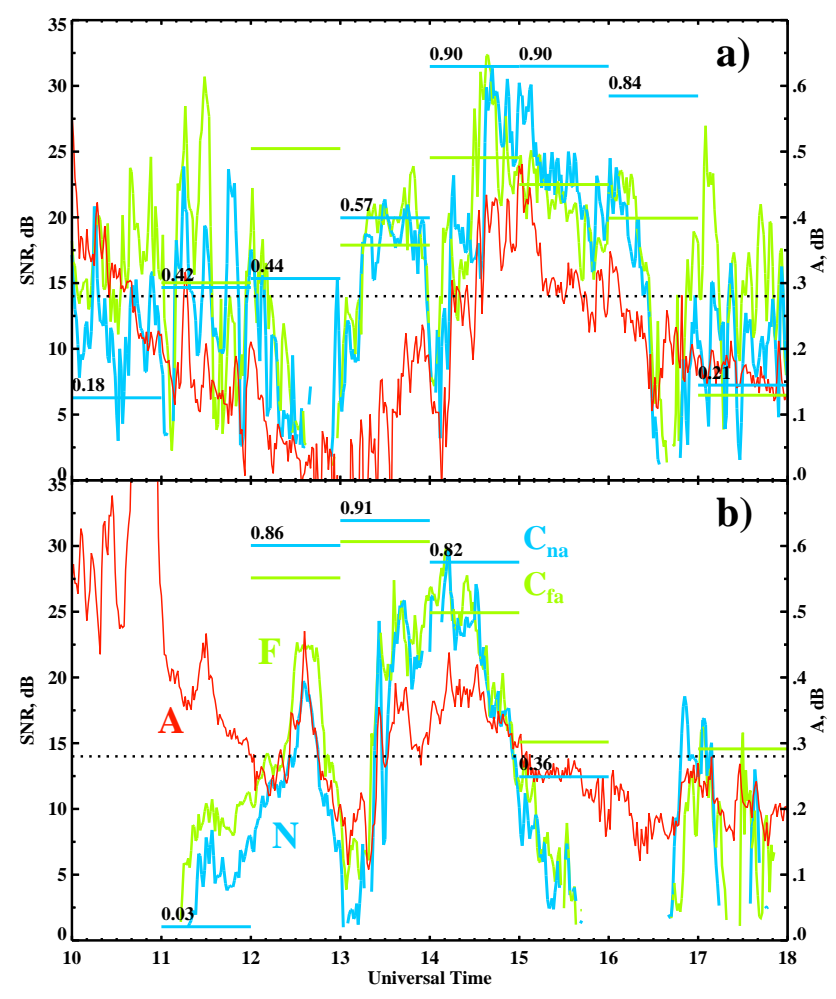

Fig. 2. Cosmic noise absorption (CNA) in IRIS beam 41 at 1-min resolution for (a) 12 and (b) 14 February 2000, 10:00-18:00 UT (red line). Backscatter power (SNR) of the STARE Norway (Finland) echoes in the closest cell (see Fig. 1) is shown by the blue (green) line. The correlation coefficients between CNA and SNR of the STARE Norway and Finland echoes $\left(C_{n a}\right.$ and $\left.C_{f a}\right)$ for each 1-h period are shown by the blue (green) horizontal lines, respectively, with the digits above and height from the bottom of each panel showing the amount of correlation. The 0.4 levels are shown by the dotted horizontal lines.

as indicated on the right axis ( 1 January is at the bottom of the diagram while 31 December is at the top). The HCPs were observed more frequently in the afternoon (12:00-17:00 UT) and during the winter (November-February). The secondary maxima were observed near midnight (21:00-02:00 UT) and during the summer (August). The heavy line shows the total (for each hour) HCP number versus time of day, normalized to the total (for all events) HCP number. Thus, during 2000-2001 only 200 periods with high correlation were observed, with the maximum occurrence in the afternoon (14:00-15:00 UT).

This conclusion, however, needs to be verified since, unlike riometer absorption data that was available most of the time ( $>98 \%$ at beam 41 in our observations), the SNR data was not, and Fig. 3 shows the histograms of the STARE Norway and Finland echo occurrence that also exhibit broad maxima in the afternoon at 14:00-17:00 UT, so that a daytime maximum in the HCP occurrence could be simply a result of the oversampling by the STARE radars. One can, however, recognize in Fig. 3 a feature that suggests otherwise, namely that the relative (with respect to the STARE 


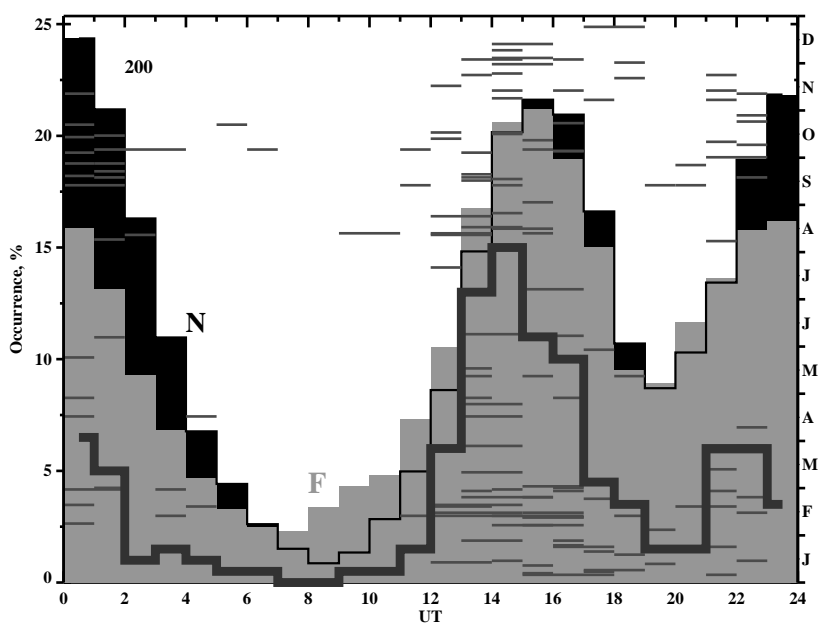

Fig. 3. Diurnal variation of the STARE echo occurrence in the cells closest to the IRIS beam 41 for 2000-2001 with the black (grey) rectangles/line showing the Norway (Finland) echo occurrence. Each 1-h period of high CNA/SNR correlation $\left(C_{n a}, C_{f a}>0.4\right)$ is shown by the horizontal line with the distance from the bottom corresponding to the time of the year, as shown on the right axis. The heavy line shows the diurnal variation of the total (for each 1-h interval) number of high correlation periods (HCPs) normalized to the total (for all $24 \mathrm{~h}$ ) number of HCPs shown in the top left corner.

echo number) HCP number variation also maximizes in the afternoon, at around 13:00-14:00 UT, when the heavy line is the highest relative to both Norway and Finland occurrences. The same effect can also be recognized if one compares the occurrences in the afternoon and midnight sectors. Both STARE echo occurrences are comparable in these two sectors, whereas the HCP occurrence is at least 2 times higher in the afternoon (15\% versus $6 \%$ ).

A similar feature is also present if one considers not only the total number of echoes versus the time of the observations, as in Fig. 3, but also the total number of 1-h periods with at least $60 \%$ echo occurrence, which is perhaps a more proper measure for the STARE echo occurrence, since this was the criterium for the correlation coefficient calculations. Figure 4 shows the $60 \%$ STARE echo occurrence (black histogram) and the relative HCP occurrence (grey thick line). To obtain the latter we divided the HCP occurrence (white line in Fig. 4) if the latter was above a critical value of 2, by the STARE occurrence and normalized it, so that the total area under the grey line is equal to $100 \%$. The STARE echo distribution in Fig. 4 is very similar to that in Fig. 3, with the maximum at 14:00-16:00 UT. The relative HCP occurrence is maximized somewhat earlier, at 12:00-15:00 UT. One can conclude that the maximum of the HCP occurrence in the afternoon sector is not purely due to the bias in the STARE echo occurrence but rather suggestive of a general pattern.

Given this pattern for the diurnal variation of HCP occurrence it is instructive to compare it with the diurnal variation for various absorption intensity levels, in attempt to reveal the typical absorption intensities during the periods with

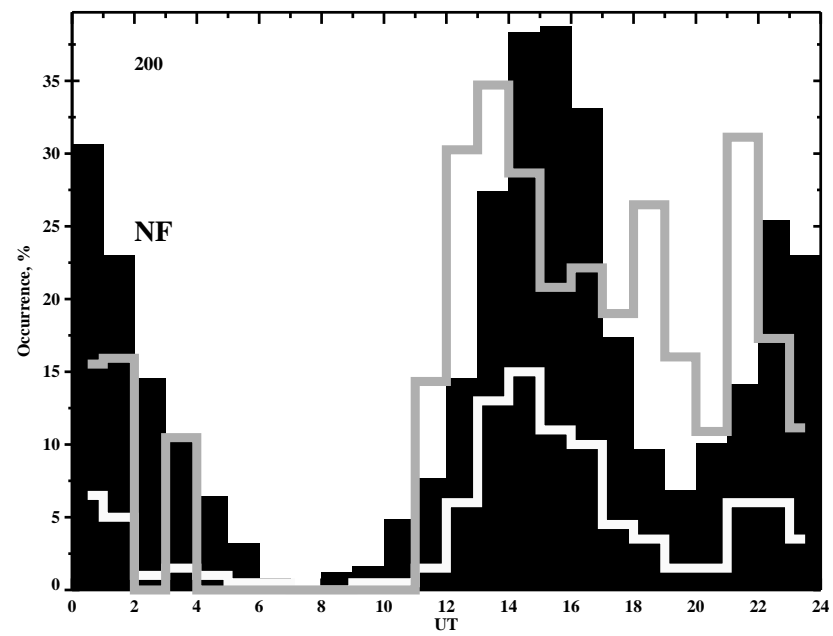

Fig. 4. Diurnal variation of the 1 -h periods of STARE observations when both radars detected echoes at least $60 \%$ of the time. The white line is the diurnal variation of the total (for each 1-h interval) number of high correlation periods (HCP) (same as in Fig. 3) and the heavy grey line is the relative (with respect to the STARE 60\%occurrence) HCP occurrence normalized to $100 \%$.

high correlation; Fig. 5 shows this comparison for (a) all 731 days of observations and (b) 111 days with at least one HCP present during 2000-2001. The absorption levels shown in Fig. 5 are computed for 1-h average absorption. The white lines in Fig. 5 are the HCP occurrence and the black lines are the relative occurrence of the $0.25<A<0.50 \mathrm{~dB}$ level with respect to the one of $0.00<A<0.25 \mathrm{~dB}$. The latter was plotted in panel (b) only when the HCP occurrence exceeded $10 \%$.

Let us first discuss the features of panel (a). Only the first distribution $(0.00<A<0.25)$ exhibits similar variation to the $\mathrm{HCP}$ occurrence, all other diurnal variations are rather anticorrelated with that of the HCPs. The relative occurrence between the two absorption levels also shows some anticorrelation, although its minimum is not exactly simultaneous with the maximum of the HCP variation but shifted by $1-$ $2 \mathrm{~h}$. Overall, absorption between $0.00-0.25 \mathrm{~dB}$ is observed more frequently in the afternoon, both in absolute terms and relative to the absorption between $0.25-0.50 \mathrm{~dB}$. During 111 days with HCPs, panel (b), the absorption is predominantly between $0.00-0.25 \mathrm{~dB}$ with some contribution from the 0.25 $0.50 \mathrm{~dB}$ level. The trends are similar to the HCP occurrence. The relative occurrence of $0.25-0.50 \mathrm{~dB}$ level is, however, somewhat greater than that for all 731 days.

In summary, the absorption was predominantly in the range $0.00-0.25 \mathrm{~dB}$ in the afternoon when the HCP occurrence was at maximum, which suggests that HCPs are associated with the low absorption intensities.

\subsection{Scatter plots of CNA/SNR correlation}

The question of what are the typical conditions for the HCPs to be observed is a fundamental one, and in this section we address the issue by plotting the correlation coefficients $C_{n a}$ 


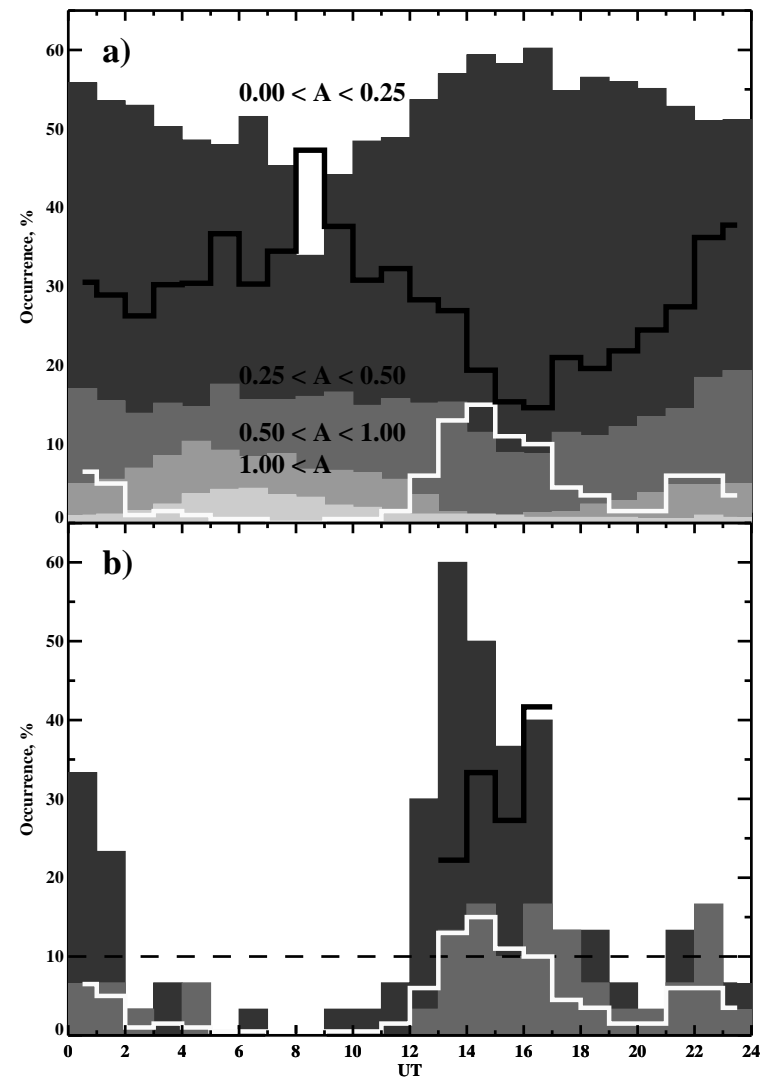

Fig. 5. The occurrence of various absorption levels for (a) all 731 days and (b) 111 days with at least one 1-h HCP during 2000-2001. The white line shows the HCP occurrence (the same as in Figs. 3 and 4) and the black line shows the occurrence of the $0.25<A<0.50$ level relative to the one of $0.0<A<0.25$.

and $C_{f a}$ against each other and colour-coding all the points in correlation coefficient between the STARE powers $C_{n f}$ and absorption intensity $A$.

Figure 6 presents the results of this comparison for the entire range of correlation coefficients from -1.0 to +1.0 and for all 111 days during which at least one $\mathrm{HCP}$ was observed. In panel (a), although the scatter is large, the points are clustered around the dotted line of ideal coincidence. Interestingly, when the discrepancies are large, for example, when $C_{n a}$ and $C_{f a}$ are of the opposite sign, $C_{n f}$ is predominantly negative (diamonds). The points also tend to be closer to the ideal coincidence line when $C_{n f}$ is larger. This point is made clearer in panel (b) which shows only the points with $C_{n f}>$ 0.8 . The mean correlation coefficients $\left(C_{n a}, C_{f a}\right)$ shown by the large black dot in panels (a) and (b) are also greater in panel (b) and so is the percentage of HCPs, $N_{h c}$. The latter are calculated by dividing the number of HCPs (points in the right top corners $n_{h c}, C_{n a}, C_{f a}>0.4$ ) by the total number of points $n_{\text {all }}$. The points with greater $C_{n f}$ are associated with greater $C_{n a}, C_{f a}$ (red points concentrate in the top-right corner). Figures $6 a, b$ thus indicate that the amount of correlation between absorption and VHF echo power depends strongly upon the amount of correlation between backscatter powers

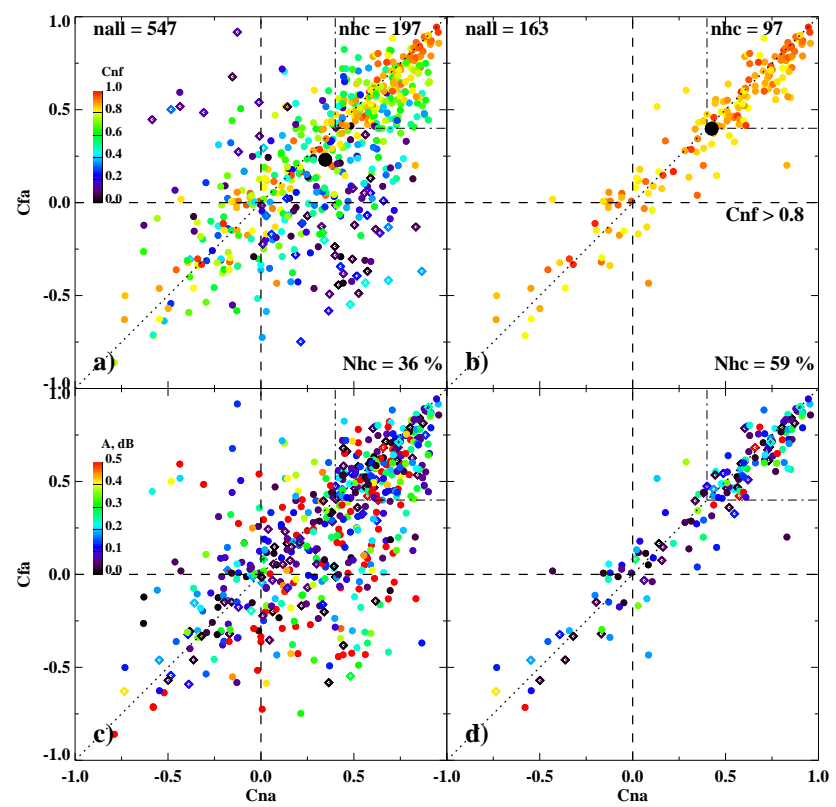

Fig. 6. The scatter plot of correlation coefficients $\left(C_{f a}\right.$ versus $\left.C_{n a}\right)$ for (a) and (c) all correlation coefficients between STARE Norway and Finland backscatter powers $C_{n f}$ and (b) and (d) for $C_{n f}>0.8$. All points in panels (a),(b) and (c),(d) are colour-coded in $C_{n f}$ and absorption intensity $A$, with the colour bar shown in the top-left corner of panels (a) and (c), respectively. The points corresponding to the positive (negative) $C_{n f}$ and $A$ are marked with circles (diamonds). The total number of all points $n_{\text {all }}$ (points with sufficiently high correlations $n_{h c}$ ) is shown in the top-left (top-right) corner with percentage of the HCPs $N_{h c}=n_{h c} / n_{\text {all }}$ shown in the bottom-right corner of panels (a) and (b). The large black circles in panels (a) and (b) mark the mean $\left(C_{n a}, C_{f a}\right)$.

recorded by the two radars. It is also noteworthy that Figs. $6 \mathrm{a}$ and $b$ do not have any noticeable bias towards either of the STARE radars; the scatter plots are fairly symmetric with respect to the ideal coincidence line and the mean correlation coefficient is only slightly larger for Norway radar. The discrepancy is larger for all $C_{n f}$, Fig. 6a (mean $C_{n a}=0.35$ versus mean $C_{f a}=0.23$ ), but this is almost entirely due to the points with negative $C_{n f}$ and $C_{f a}$ and positive $C_{n a}$ (cluster of diamonds in the bottom-right quadrant), so that for $C_{n f}>0.8$ (Fig. 6b) the discrepancy becomes smaller ( 0.43 versus 0.40$)$.

Panels (c) and (d) present the same data as panels (a) and (b), except that colour-coding has been done in absorption intensity. There is no clear trend in the absorption in panels (c); all points are rather mixed. The points with larger absorption tend to appear farther from the ideal coincidence line, so that in panel (d) the points near it predominantly have small absorption, with exception of the top-right corner.

\subsection{CNA/SNR correlation and spatial effects}

In the beginning of Sect. 3 we noted that the observations at the IRIS beam 41 had a better opportunity to show high correlation than the ones at beam 9 , simply because of the spatial 


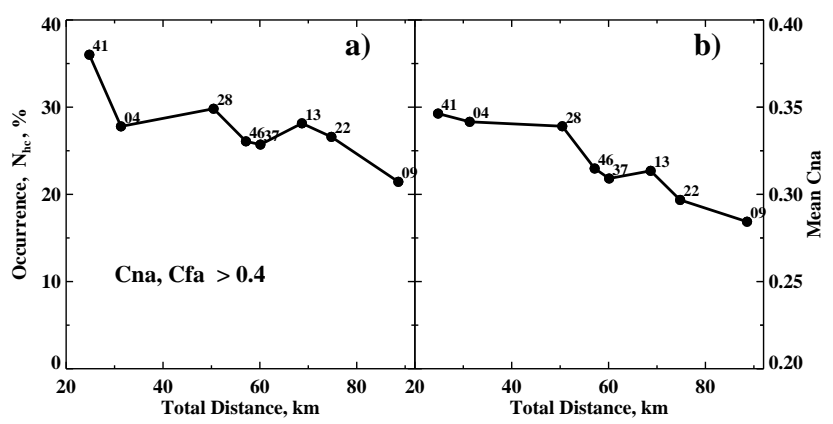

Fig. 7. Dependence of the (a) occurrence of HCPs and (b) mean correlation coefficient $C_{n a}$ upon the total distance between the collecting area foci for selected IRIS beams. The IRIS beam numbers are shown by digits near each point.

differences in observational areas. In this section we explore the spatial effects by employing the data from all considered IRIS beams and corresponding STARE radar cells. To characterize the differences in observational areas we introduce a simple quantitative measure, namely for each of the eight IRIS beams we consider the total distance between the points of maximum sensitivity, that is the perimeter of the triangle formed by the centers of the two radar cells and by the central position of the IRIS beam. This parameter describes the degree of similarity between the observational areas, both in terms of the orientation and foci closeness; the closer it is to zero, the larger the similarity. Figure 7 shows the (a) occurrence of HCPs and (b) the mean correlation coefficient $C_{n a}$ as functions of the total distance. The occurrence $N_{h c}$ and mean coefficient $C_{n a}$ are computed as described in the previous section. The IRIS beam number is indicated near each point.

As one would expect, point 41 is the highest in both panels having the largest HCP occurrence and mean correlation, while point 9 is the lowest. Overall, there is a decrease in both HCP occurrence and mean correlation with total distance increase, which indicates that the spatial effects contribute strongly to the amount of correlation observed.

One can argue that Fig. 7 is biased towards IRIS beam 41 , since the event selection was based entirely on the data from this direction. We therefore repeated the HCP occurrence calculations seven more times (by taking as the "base" direction IRIS beams 4, 28, 46, 37, 13, 22, and 9, selecting corresponding event databases, and computing $N_{h c}$ for each of these directions) and found only marginal differences as compared to the results presented in Fig. 7. Thus, the HCP respective occurrences were $36,27,29,26,25,28,26$, and $21 \%$, where the first value refers to the HCP occurrence at beam 41 from Fig. 7 a.

\section{Discussion}

The observations of high positive correlation between the riometer absorption and the VHF echo power reported in this paper are the first observations of this kind, which necessitates the consideration of this phenomenon in the context of other auroral phenomena, as outlined in Sect. 1. In this sense of special importance is the fact that a vast majority of high correlation events occur in the afternoon sector, between 12:00 and 19:00 UT, Fig. 3. On a case-by-case basis, the events of very long duration and high correlation were also observed between 12:00 and 17:00 UT, Fig. 2. The absorption events in this time sector are significantly less understood than other absorption types such as, for example, midnight and morning absorption, both linked to the substorm activity on the nightside (Stauning, 1996a). It is, therefore, not surprising that the high correlation was observed away from the midnight meridian, in sharp contrast with the results for the correlation with the optical intensity (Ansari, 1964) and magnetic disturbance (Brown and Campbell, 1962), since the physical mechanism responsible for the enhanced CNA levels could easily be different for the afternoon events.

It is well accepted that the bulk of the absorption events is associated with the enhanced ionization (ambient electron density $N_{e}$ ) at D-/lower E-region altitudes caused by the precipitating electrons (Hargreaves, 1969; Stauning, 1996a). Some of the absorption events observed with riometers in the polar cap, however, have been also associated in the past with an increase in the E-region collision frequencies caused by electron heating (Stauning, 1984; Stauning and Olesen, 1989) and with an increase in F-region densities (Rosenberg et al., 1993; Wang et al., 1994).

The power of coherent echoes is, on the other hand, a complex function, depending upon the electric field intensity $E$ (through the fractional electron density fluctuation amplitude $\delta N_{e} / N_{e}$ ), ambient electron density $N_{e}$, angle between the direction of propagation $\boldsymbol{k}$ and that of the magnetic field $\boldsymbol{B}$ (aspect angle $\alpha$ ), angle between $\boldsymbol{k}$ and the plasma drift vector $\boldsymbol{V}_{d}$ (flow angle $\theta$ ), and distance from the radar site to the backscatter region $r$ (e.g. Haldoupis, 1989). In this study, we consider observations at essentially one observational point (one beam of IRIS and closest STARE cells), which greatly simplifies the problem, as both $r$ and $\alpha$ can be assumed to be constants with refraction negligible at $140 \mathrm{MHz}$. Even though $r$ and $\alpha$ are different for the two radars, this is not an issue since we consider the correlations. The flow angle effects, in principle, could be present in the SNR data, as the echo power is known to decrease with the deviation from the plasma drift direction (André, 1983). We are not in a position to estimate the flow angles and to study these effects rigourously, since this would require the information on the electric field at the locations of the selected IRIS beams during the entire period under investigation. Given that the interpretation of the STARE velocity data is far from straightforward, in particular, during the intervals of strong plasma flow (Uspensky et al., 2004), we focused on the echo power data. First of all, we were interested in the fact of a high CNA/SNR correlation itself and were confident that the usage of the relatively short $1-\mathrm{h}$ intervals minimized the flow angle effects in the correlation analysis. In addition, the 
magnetic perturbation data from the International Monitor for Auroral Geomagnetic Effects (IMAGE) and Sub-Auroral Magnetometer Network (SAMNET) fluxgate magnetometers for individual events showed that the electrojet flow was relatively stable in terms of direction. We think, therefore, that the most important parameters in the context of this study are the electric field intensity $E$ and the electron density $N_{e}$. The relative contribution of $E$ and $N_{e}$ to the power of coherent echoes is still not well understood though, with some studies showing a strong electric field control (e.g. Haldoupis et al., 1984) and others suggesting that power is proportional to $N_{e}^{2}$ (Uspensky et al., 1983; Starkov et al., 1983; Haldoupis et al., 1990).

The combination of high electric fields and electron densities thus may be the reason for the enhancements in both auroral absorption and VHF echo power, which can explain our observations of high correlation. There is one drawback in this interpretation though, namely that the principal absorption layer is somewhat lower than the typical altitude range where VHF radars detect irregularities (85-95 km for absorption versus $105-110 \mathrm{~km}$ for irregularities). This is not a problem if the densities vary to a large degree synchronously for the altitude range in question $(85-110 \mathrm{~km})$, which could be the case if the spectra of the precipitating electrons are far from being monoenergetic. The wealth of existing observational evidence on the correlation between optical intensity and CNA seems to support this hypothesis, since optical measurements typically refer to higher altitudes than those of absorption (e.g. Holt and Omholt, 1962; Ansari, 1964).

To check whether the electrons precipitating at two set of heights (around 90 and $110 \mathrm{~km}$ ) belong to the same population, one would need to compare the electron flux measurements for two corresponding energy ranges. The empirical formula for the effective altitude of the energy deposition derived by del Pozo et al. (1997), $\varepsilon(H)=4 \times 10^{5} \exp (-0.101 H)$, gives electron energies of 45 and $6 \mathrm{keV}$, respectively. Unfortunately, the electron flux measurements in both energy ranges are difficult to obtain; the instruments on board of the geostationary satellites, such as Los Alamos National Laboratory (LANL) satellites, measure the fluxes of the electrons in the inner magnetosphere with $\varepsilon>50 \mathrm{keV}$, whereas ionospheric satellites, such as Defense Meteorological Satellite Program (DMSP), can provide the fluxes of precipitating electrons with $\varepsilon<32 \mathrm{keV}$. The former give temporal variation at several points in the region of closed field lines ( $6.6 \mathrm{R}_{E}$, where $\mathrm{R}_{E}$ is the Earth's radius) while the latter provide spatial variation along several satellite paths (Sun-synchronous orbits at $\sim 830$-km altitude). The two lowest electron energy channels for a Synchronous Orbit Particle Analyzer (SOPA) instrument (Belian et al., 1992) on board of the presently operational LANL satellites are 50-75 and 75-105 keV. Although these energies are somewhat greater than those of interest, one can check whether the electron fluxes were synchronous, and in Fig. 8 we show the electron flux measurements from these two energy channels from the LANL satellite 1994-084, the satellite closest in magnetic local time (MLT $\sim \mathrm{UT}+10)$ to the IRIS viewing

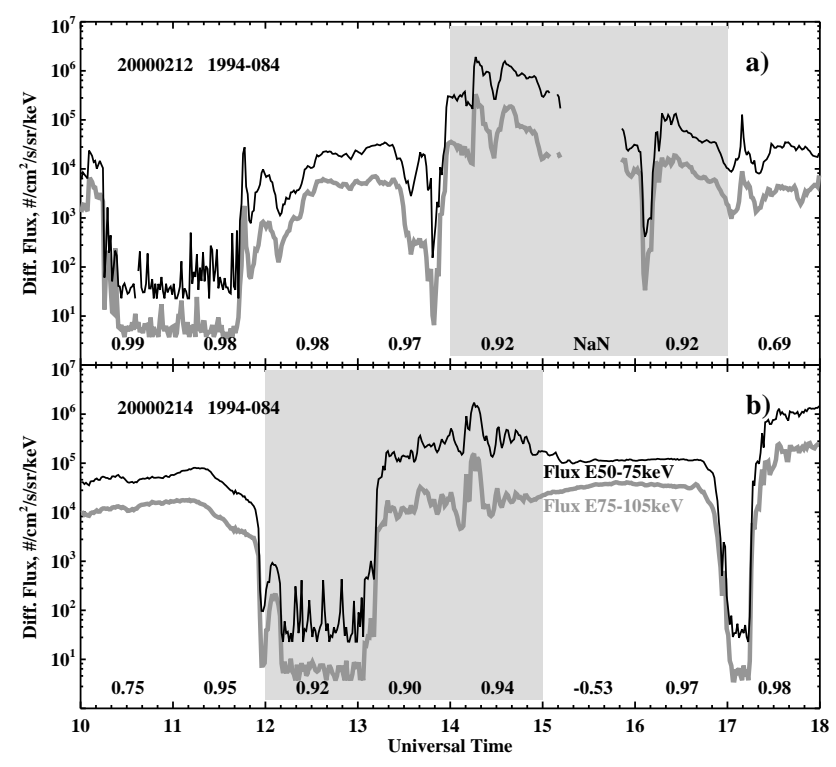

Fig. 8. SOPA spin-averaged electron flux measurements at 1-min resolution for (a) 12 and (b) 14 February 2000, 10:00-18:00 UT. The highlighted areas correspond to the periods of very high correlation from Fig. 2. The digits at the bottom of each panel show the correlation coefficients between the fluxes at two lowest energy channels for each 1-h interval.

area (MLT $\sim \mathrm{UT}+3$ ), for the same periods as in Fig. 2.

The flux variations for the two lowest channels are well synchronized, meaning that the electrons at the lowest end of energy spectrum in the inner magnetosphere belong to the same population, which, in turn, suggests that the fluxes of precipitating electrons are likely to vary synchronously, as well. The periods of very high CNA/SNR correlation from Fig. 2 (highlighted in Fig. 8) correspond to the intervals during which the correlation between fluxes was very high (above 0.9). One can conclude that the electron flux data from the LANL satellite is consistent with the idea that the CNA/SNR correlation is due to the electrons whose fluxes vary synchronously, to a first approximation, within a range of altitudes (90-110 km).

Another possibility is that the CNA/SNR correlation is associated with the electrons precipitating at essentially the same altitude range, since the division between the typical altitudes for CNA and SNR measurements is by no means well defined. The thickness of the principal absorption layer (vertical distance between points where absorption is half that at the peak) can be as high as $15-20 \mathrm{~km}$ (e.g. Jespersen et al., 1968) and the absorption layer peak can be well above $90 \mathrm{~km}$ (e.g. Terkildsen et al., 2004). The VHF backscatter signal, on the other hand, can come from the wide range of heights with the altitude range which contributes the most to the backscatter power that is dependent upon the background density $N_{e}(h)$ and fractional density fluctuation amplitude $\left(\delta N_{e} / N_{e}\right)(h)$ profiles, as described recently by Uspensky et al. (2003). 
One particular issue needs to be discussed in this context, namely the nature of VHF echoes observed during the HCPs or, more specifically, whether these echoes were in fact polar mesospheric echoes, since in the past a positive CNA/SNR correlation has been reported for PMSE observations (e.g. Czechowsky et al., 1989; Kirkwood et al., 1995; Barabash et al., 2002). One should bear in mind, however, that the vast majority of the PMSE observations have been performed with vertical incidence VHF radars at frequencies near $50 \mathrm{MHz}$, while our measurements were carried out with an oblique-sounding 144-MHz radar, sensitive to fieldaligned irregularities in the $\mathrm{E}$ region. For a similar experiment geometry, the short-duration $(<20 \mathrm{~min})$ PMSE echoes detected by the Super Dual Auroral Radar Network (SuperDARN) Syowa East radar, at very short ranges (180$315 \mathrm{~km}$ ), have been reported recently by Hosokawa et al. (2004). In our observations the ranges were much larger $(>700 \mathrm{~km})$ and the VHF backscatter was typically observed as wide $(\sim 400 \mathrm{~km})$ and relatively stable $(1-2 \mathrm{~h})$ bands of echoes; these features are typical for the E-region echoes as opposed to the polar mesospheric echoes.

The results on the diurnal/seasonal variation presented in this study also disagree with the PMSE hypothesis. The HCP occurrence was clearly maximized in the winter, while PMSEs are observed from May to August in the Northern Hemisphere (Bremer et al., 2003). Mesospheric echoes are observed also in the winter but these echoes are significantly less frequent and are typically associated with the enhanced particle precipitation (Czechowsky et al., 1989). Our result on the HCP diurnal variation differs from that of Barabash et al. (2002), who reported a significant correlation between the square of CNA and PMSE intensities in the 20:00-06:00 UT sector. We conclude, therefore, that VHF echoes in our observations are unlikely to originate from the D-region.

Diurnal variation of HCP was similar to that of the lowest absorption level $(0.0-0.25 \mathrm{~dB})$ and different from other higher levels of absorption, Fig. 5a. For days with at least one HCP, the CNA at the highest levels $(>0.5 \mathrm{~dB})$ was virtually negligible, Fig. $5 b$. These features suggest that HCPs are associated with the softer electrons that precipitate at higher E-region altitudes and produce relatively small (though still noticeable) absorption enhancements. The lower the electron energy, the larger the altitude of maximum ionization and hence the better the chances for high correlation with the backscatter power. If so, one would expect the absorption intensity to decrease with a correlation coefficient increase. Indeed, for individual events this was often the case, for example, in Fig. 2 the highest correlation was observed when CNA was at maximum. Statistically, however, the effect was not obvious; in Figs. $6 \mathrm{c}$ and d points with larger absorption were scattered in a seemingly random fashion. We believe that the reason can be that, apart from the physical conditions (e.g. particle energy which stipulates the maximum ionization height) at a specific point of measurement, the amount of correlation is strongly controlled by the degree of spatial homogeneity, as discussed below.
The question that we address is: How much of the correlation is "lost" due to the differences in observational areas, as very often riometer absorption and other parameters are compared at locations that are somewhat apart from each other? In this study we specifically looked at the problem by exploiting a unique opportunity stemming from the experiment geometry in Northern Scandinavia, where riometer absorption measurements with high spatial resolution can be combined with the stereoscopic measurements by two VHF radars.

We first looked at the effects of the correlation between echo powers measured by two radars which can be considered as a measure of spatial homogeneity/inhomogeneity, as explained below. The radars monitor slightly different locations and, in addition, collect a backscatter signal from slightly different areas (radar cells), Fig. 1. In a situation when the correlation $C_{n f}$ is high, the effects arising from these differences are not significant because of the general spatial homogeneity of processes associated with the backscatter signal formation. Thus, if the generation of irregularities is spatially homogeneous in the common area that includes both Norway and Finland radar cells, one can expect that the echo power would be the same for both radars as well, and so would the correlation between some other parameter (CNA in our case) and SNR, in agreement with Fig. 6b. For events with sufficiently high correlation coefficients $C_{n f}$, the agreement between CNA/SNR correlation coefficients is greatly improved. The increases in the mean correlation coefficients, $C_{n a}$ and $C_{f a}$, and in the HCP percentage, $N_{h c}$, were also significant, Fig. 6 . We hence conclude that the CNA/SNR correlation is dependent upon the spatial homogeneity of the processes associated with cosmic noise absorption and backscatter power formation.

The data presented in Fig. 6, however, also indicate that the percentage of the events with high correlation $C_{n f}$ is rather low (yellow/red dots in Fig. 6 are less frequent than black/blue/green ones) and that very often it is negative, so that more often than not the backscatter signal formation process is inhomogeneous. In this situation a similarity between the collection areas becomes an important factor which can significantly affect the amount of CNA/SNR correlation observed. Indeed, Fig. 7 shows that this is exactly the case. Both the HCP occurrence $N_{h c}$ and the mean correlation $C_{n a}$ are reduced if the distances between the collection area foci are large, which we interpret as a decrease associated with differences in observational areas, combined with considerable spatial inhomogeneity.

In our discussion of the factors responsible for the CNA/SNR correlation we emphasized the importance of both electric field intensity and ambient electron density but left unaddressed the issue of the dominant factor, since no direct measurements of these two parameters were available. The HCPs were predominantly observed in the afternoon sector in between 15 and 20 MLT, Fig. 3. Given the latitude of the common FoV ( $\sim 66^{\circ}$ MLAT), the observations in this sector predominantly refer to the eastward electrojet or to the earthward flow region in the magnetospheric plasma sheet. In this 
region, the electrons could be accelerated by the earthward convection to sufficiently high energies to be detected by a riometer on the Earth's surface. If so, one can expect a certain amount of correlation between riometer absorption and plasma drift velocity. Even though the derivation of the full plasma drift vector from the STARE Doppler velocity measurements could be quite challenging, as demonstrated recently by Uspensky et al. (2003, 2004), one can still attempt to identify the effect sought in the Doppler velocity data. Figure 9 shows the results of the analysis analogous to that of Fig. 6, except that the points are colour-coded in correlation coefficient between the STARE Norway velocity and IRIS absorption, $C_{v n a}$. One can recognize in Fig. 9a that, indeed, green and yellow points cluster in the top-right corner, meaning that the periods with very high $C_{n a}>0.8$ also have high $C_{v n a}=0.6-0.8$. The same is true for Fig. $9 \mathrm{~b}\left(C_{n f}>0.8\right.$, "homogenous case"), which, together with the fact that in Figs. 6a and b and Figs. 9a and b points with high $C_{n f}$ and $C_{v n a}$ belong to two different (although intersecting) populations, suggests that there are two different factors involved. One can also see from Fig. 9 that not all HCPs are associated with a high correlation between CNA and the STARE velocity, and that the amount of correlation for velocity is lower than that for power. One can conclude that for some HCP events, especially for ones that exhibit a very high correlation, the electric field seems to dominate, which is consistent with the "electron acceleration by a convection" scenario proposed. More studies are needed, however, in order to make a more definite conclusion, in particular, those that would utilize the in-situ observations of the magnetospheric particle dynamics and ground-based measurements of the electron density.

\section{Summary}

The analysis of the data collected during 2 years of operation in the common area between $7 \times 7$-beam imaging riometer and two VHF coherent radars in Northern Scandinavia has revealed $\sim 200$ one-hour intervals (during 111 days) of statistically significant correlation between the cosmic noise absorption and the signal-to-noise ratio. During several events the CNA/SNR correlation was particularly high $(>0.8)$ for up to $3 \mathrm{~h}$. The high correlation periods are observed predominantly in the afternoon sector and in the winter, with the secondary peaks near midnight and in the summer. The diurnal variation of HCPs is similar to that for events with 1-h mean absorption between 0.0 and $0.25 \mathrm{~dB}$. Consideration of all 1-h intervals for 111 days with HCPs present, on the other hand, did not reveal any trend in the correlation coefficient dependence upon the absorption intensity. The CNA/SNR correlation at a given observational point was found to depend strongly upon the correlation between the coherent echo intensities measured by two VHF radars at the same location; both the CNA/SNR correlation and agreement between the CNA/SNR correlation for the two radars was greatly reduced when the correlation between the echo powers mea-

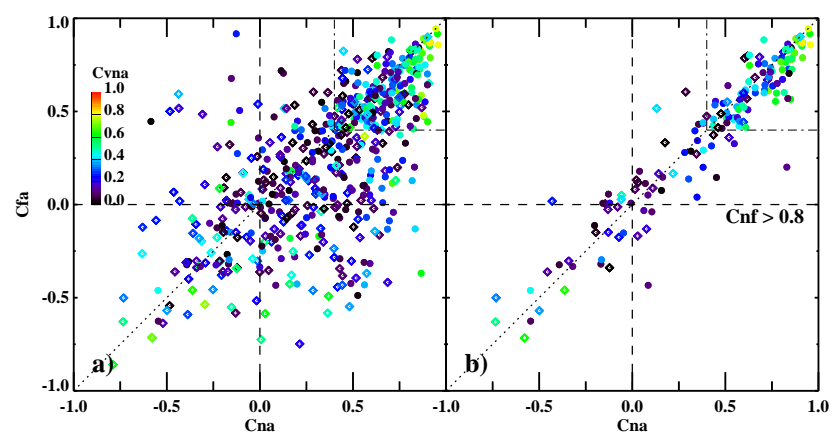

Fig. 9. The same as Figs. $6 \mathrm{a}$ and $\mathrm{b}$ but colour-coded in correlation coefficient between the STARE Norway velocity and IRIS absorption, $C_{v n a}$.

sured by the two radars was smaller. From a comparison between measurements at 8 observational directions, the HCP occurrence and mean correlation coefficients decrease as the total distance between points of maximum sensitivity (foci) increases; both parameters are maximized for measurements at a direction for which the intersection of the riometer beam with the ionosphere and radar cell are most comparable in terms of spatial coverage, orientation, and proximity between the collection area foci.

\section{Conclusions}

The events that exhibit a statistically significant correlation between the cosmic noise absorption and the VHF coherent echo intensity, while being relatively rare, show consistent features in their occurrence and correlation amount, from which the following conclusions can be drawn.

1. There is evidence that high correlation periods are associated with the electrons in the lower end of the energy spectrum precipitating at the E-region bottom side as opposed to more energetic particles with the maximum ionization altitude in the D-region. The echoes detected by an oblique-sounding VHF radar during HCPs are unlikely to be mesospheric echoes. These echoes represent the scatter from the E-region field-aligned irregularities. Highly correlated variations in CNA and SNR are likely to be related to variation in ionization at two sets of heights caused by the precipitating electrons at two close sets of energies whose fluxes are well correlated.

2. The amount of correlation between riometer absorption and VHF echo power is strongly controlled by the degree of spatial homogeneity of the processes leading to enhanced CNA and SNR.

3. For riometer beams and radar cells comparable in areas and shapes, the correlation is reduced as the areas become less overlapped (e.g. due to rotation) and as the distance between points of maximum sensitivity increases. It is therefore imperative to employ the CNA 
and SNR measurements in similar radio signal collection areas when comparing these parameters (e.g. in studies of the factors controlling CNA and SNR).

Acknowledgements. This research was supported by the Particle Physics and Astronomy Research Council, UK (research grant PPA/G/S/2001/00491). The IRIS facility is operated by the Department of Communication Systems at Lancaster University and funded by PPARC in collaboration with the Sodankylä Geophysical Observatory. The STARE system is operated jointly by the Max Plank Institute for Aeronomie, Germany, and the Finnish Meteorological Institute, Finland, in cooperation with SINTEF, University of Trondheim, Norway. The IMAGE magnetometer data are collected as a Finnish-German-Norwegian-Polish-Russian-Swedish project conducted by the Technical University of Braunschweig and FMI. The SAMNET magnetometers are funded by PPARC. We are grateful to M. Uspensky and P. Janhunen of FMI for providing access to the STARE data and to D. DeLapp and G. Reeves of Los Alamos National Laboratory for providing the SOPA LANL particle flux data.

Topical Editor W. Kofmann thanks M. J. Jarvis and E. Nielsen for their help in evaluating this paper.

\section{References}

André, D.: The dependence of the relative backscatter cross section of 1-m density fluctuations in the auroral electrojet on the angle between electron drift and radar wave vector, J. Geophys. Res., 88, 8043-8049, 1983.

Ansari, Z. A.: The aurorally associated absorption of cosmic noise at College, Alaska, J. Geophys. Res., 69, 4493-4513, 1964.

Barabash, V., Kirkwood, S., and Chilson, P. B.: Are variations in PMSE intensity affected by energetic particle precipitation?, Ann. Geophys., 20, 539-545, 2002,

SRef-ID: 1432-0576/ag/2002-20-539.

Belian, R. D., Gisler, G. R., Cayton, T., and Christensen, R.: High$\mathrm{Z}$ energetic particles at geostationary orbit during the great Solar Proton Event series of October 1989, J. Geophys. Res., 97, 16 897-16906, 1992.

Bremer, J., Hoffman, P., and Hansen, T. L.: Geomagnetic control of polar mesosphere summer echoes, Ann. Geophys., 18, 202-208, 2000 ,

\section{SRef-ID: 1432-0576/ag/2000-18-202.}

Bremer, J., Hoffmann, P., Latteck, R., and Singer, W.: Seasonal and long-term variations of PMSE from VHF radar observations at Andenes, Norway, J. Geophys. Res., 108, doi:10.1029/2002JD002369, 2003.

Brown, R. R. and Barcus, J. R.: Balloon observations of the extent and structure of auroral zone electron precipitation events, J. Geophys. Res., 68, 6069-6077, 1963.

Brown, R. R. and Campbell, W. H.: An auroral-zone electron precipitation event and its relationship to a magnetic bay, J. Geophys. Res., 67, 1357-1366, 1962.

Browne, S., Hargreaves, J. K., and Honary, B.: An imaging riometer for ionospheric studies, Electronics and Communication, 7, 209217, 1995.

Czechowsky, P., Reid, I. M., Ruster, R., and Schmidt, G.: VHF radar echoes observed in the summer and winter polar mesosphere over Andoya, Norway, J. Geophys. Res., 94, 5199-5217, 1989. del Pozo, C. F., Hargreaves, J. K., and Aylward, A. D.: Ion composition and effective ion recombination rate in the nighttime auroral lower ionosphere, J. Atmos. Sol. Terr. Phys., 59, 1919-1943, 1997.

Detrick, D. L. and Rosenberg, T. J.: A phased-array radiowave imager for studies of cosmic noise absorption, Radio Sci., 25, 325338, 1990.

Greenwald, R. A., Weiss, W., Nielsen, E., and Thomson, N. R.: STARE: A new radar auroral backscatter in Northern Scandinavia, Radio Sci., 13, 1021-1029, 1978.

Haldoupis, C.: A review on radio studies of auroral E region ionospheric irregularities, Ann. Geophys., 7, 239-258, 1989.

Haldoupis, C., Nielsen, E., and Ierkic, H. M.: STARE Doppler spectral studies of westward electrojet radar aurora, Planet. Space Sci., 32, 1291-1300, 1984.

Haldoupis, C., Nielsen, E., and Schlegel, K.: Dependence of radar auroral scattering cross section on the ambient electron density and the destabilizing electric field, Ann. Geophys., 8, 195-212, 1990.

Hargreaves, J. K.: On the variation of auroral radio absorption with geomagnetic activity, Planet. Space Sci., 14, 991-1006, 1966.

Hargreaves, J. K.: Auroral absorption of HF radio waves in the ionosphere: A review of results from the first decade of riometry, Proc. IEEE, 57, 1348-1373, 1969.

Holt, O. and Omholt, A.: Auroral luminosity and absorption of cosmic radio noise, J. Atmos. Terr. Phys., 24, 467-474, 1962.

Hosokawa, K., Ogawa, T., Yukimatu, A. S., Sato, N., and Iyemori, T.: Statistics of Antarctic mesospheric echoes observed with the SuperDARN Syowa radar, Geophys. Res. Lett., 31, doi:10.1029/2003GL018776, 2004.

Jespersen, M., Kane, J. A., and Landmark, B.: Electron and positive ion density measurements during conditions of auroral absorption, J. Atmos. Terr. Phys., 30, 1955-1964, 1968.

Kavanagh, A. J., Honary, F., McCrea, I. W., Donovan, E., Woodfield, E. E., Manninen, J., and Anderson, P. C.: Substorm related changes in precipitation in the dayside auroral zone - a multi instrument case study, Ann. Geophys., 20, 1321-1334, 2002,

SRef-ID: 1432-0576/ag/2002-20-1321.

Kavanagh, A. J., Kosch, M. J., Honary, F., Senior, A., Marple, S. R., Woodfield, E. E., and McCrea, I. W.: The statistical dependence of auroral absorption on geomagnetic and solar wind parameters, Ann. Geophys., 22, 877-887, 2004,

SRef-ID: 1432-0576/ag/2004-22-877.

Kirkwood, S., Cho, J., Hall, C. M., Hoppe, U.-P., Murtagh, D. P., Stegman, J., Swartz, W. E., van Eyken, A. P., Wannberg, G., and Witt, G.: A comparison of PMSE and other ground-based observations during the NLC-91 campaign, J. Atmos. Terr. Phys., 57, 35-44, 1995.

Klostermeyer, J.: On the diurnal variation of polar mesosphere summer echoes, Geophys. Res. Lett., 26, 3301-3304, 1999.

Nielsen, E.: The STARE system and some of its applications, pp. 213-224, In: IMS Source Book Guide to the International Magnetospheric Study Data Analysis, (Eds.) Russell, C. T. and Southwood, D. J., Washington, D.C., AGU, 1982.

Nishino, M., Nishitani, N., Sato, N., Yamagishi, H., Lester, M., and Holtet, J. A.: A rectified response of daytime radio wave absorption to southward and northward excursions during northward interplanetary magnetic field: A case study, Adv. Polar Upper Atmos. Res., 13, 139-153, 1999.

Rosenberg, T. J., Wang, Z., Rodger, A. S., Dudeney, J. R., and Baker, K. B.: Imaging riometer and HF radar measurements of drifting $\mathrm{F}$ region electron density structures in the polar cap, J. 
Geophys. Res., 98, 7757-7764, 1993.

Starkov, G. V., Oksman, J., Uspensky, M. V., and Kustov, A. V.: On the dependence of radar aurora amplitude on ionospheric electron density, J. Geophys., 52, 49-52, 1983.

Stauning, P.: Absorption of cosmic noise in the E-region during electron heating events, Geophys. Res. Lett., 11, 1184-1187, 1984.

Stauning, P.: Investigations of ionospheric radio wave absorption process using imaging riometer techniques, J. Atmos. Terr. Phys., 58, 753-764, 1996a.

Stauning, P.: High-latitude D- and E-region investigations using imaging riometer observations, J. Atmos. Terr. Phys., 58, 765783, 1996b.

Stauning, P. and Olesen, J. K.: Observations of the unstable plasma in the disturbed polar E-region, Physica Scripta, 40, 325-332, 1989.

Terkildsen, M. B., Fraser, B. J., and Yamagishi, H.: Determination of the altitudinal peak of cosmic noise absorption using a vertical parallax technique, Geophys. Res. Lett., 31, doi:10.1029/2003GL019068, 2004.
Uspensky, M., Koustov, A., Janhunen, P., Pellinen, R., Danskin, D., and Nozawa, S.: STARE velocities: importance of offorthogonality and ion motions, Ann. Geophys., 21, 729-743, 2003,

SRef-ID: 1432-0576/ag/2003-21-729.

Uspensky, M., Koustov, A., Janhunen, P., Nielsen, E., Kauristie, K., Amm, O., Pellinen, R., Opgenoorth, H., and Pirjola, R.: STARE velocities: 2 Evening westward electron flow, Ann. Geophys., 22, 1077-1091, 2004,

SRef-ID: 1432-0576/ag/2004-22-1077.

Uspensky, M. V., Pellinen, R. J., Baumjohann, W., Starkov, G. V., Nielsen, E., Sofko, G., and Kaila, K. U.: Spatial variations of ionospheric conductivity and radar auroral amplitude in the eastward electrojet region during pre-substorm conditions, J. Geophys., 52, 40-48, 1983.

Wang, Z., Rosenberg, T. J., Stauning, P., Basu, S., and Crowley, G.: Calculations of riometer absorption associated with $F$ region plasma structures based on Sondre Stromfjord incoherent scatter radar observations, Radio Sci., 29, 209-215, 1994. 